Canadian

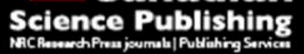

Canadian Journal of Civil Engineering Revue canadienne de génie civil

\title{
New and Improved Three and One-Third Parabolic Channel and Most Efficient Hydraulic Section
}

\begin{tabular}{|r|l|}
\hline Journal: & Canadian Journal of Civil Engineering \\
\hline Manuscript ID & cjce-2016-0535.R1 \\
\hline Manuscript Type: & Technical Note \\
\hline Date Submitted by the Author: & 18 -Jan-2017 \\
\hline Keymlete List of Authors: & $\begin{array}{l}\text { Han, Yan-Cheng; University of Jinan, College of Resources and } \\
\text { Environment } \\
\text { Easa, Said; Ryerson University, Civil Engineering }\end{array}$ \\
\hline Khree and one-third parabolic section, Open channel, Most efficient \\
\hline
\end{tabular}

\section{SCHOLARONE ${ }^{\text {m }}$}

Manuscripts 


\title{
New and Improved Three and One-Third Parabolic Channel and Most Efficient Hydraulic Section
}

\author{
Yan-Cheng $\operatorname{Han}^{1}$ Said M. Easa ${ }^{2}$ \\ ${ }^{1}$ Assoc. Prof., College of Resources and Environment, Univ. of Jinan, E-mail: \\ stu_hanyc@ujn.edu.cn \\ ${ }^{2}$ Prof., Dept. of Civ. Eng., Ryerson Univ., Toronto, ON, Canada M5B 2K3. E-mail: \\ seasa@ryerson.ca
}

Correspondence

Dr. Yan-Cheng Han

stu_hanyc@ujn.edu.cn

University of Jinan

College of Resources and Environment

No.336 West road of NanXinZhuang

Jinan

250022

China 
Abstract. Several parabolic-shaped open channel sections are available in the literature, including quadratic and semi-cubic parabolic sections. This paper presents a three and one-third parabolic cross-section that has superior characteristics compared to those of previous parabolic-shaped sections. The section characteristics, including two approximate formulae for the wetted perimeter and a simple iterative formula for the normal water depth are presented. The exact solution for the most efficient hydraulic section is derived. The results show the width-depth ratio for the most efficient hydraulic section is 2.1273 . Practical applications of the proposed most efficient hydraulic section are presented, including direct formulas for the discharge and explicit formulas of normal and critical depths. The results show that the proposed section improves the hydraulic characteristics with other parabolic sections and trapezoidal section.

Key Words: Three and one-third parabolic section; Open channel; Most efficient hydraulic section. 


\section{Introduction}

Researchers have carried out numerous efforts to develop new channel sections that maximize the discharge under the same flow area or minimize construction cost under the same discharge (Das, 2007; Easa 2016).). Existing parabolic channel sections can be divided into two categories: simple parabolic-shaped sections and complex parabolic-shaped sections. The simple parabolic-shaped sections, which have no horizontal bottom, include semi-cubic parabolic section (Han 2015), quadratic parabolic section (Loganathan 1991; Chahar 2005; Aksoy and Altan-Sakarya 2006) and cubic parabolic section (Han and Easa 2016). The complex parabolic-shaped sections, which have a horizontal bottom, include sections with semi-cubic parabolic sides (Han 2015), quadratic parabolic sides (Das 2007), and two-segment quadratic parabolic sides (Easa 2009).

The most efficient hydraulic (MEH) section has the largest flow capacity under the same flow area, and at the same time can make construction cost close to the minimum (Jain et al., 2004). It has been extensively used in channel design. For the simple sections, the semi-circular section has the largest capacity. However, the side slope of the semi-circular section at water surface is vertical and therefore the freeboard must be vertical which poses construction difficulties (Vatankhah 2014). After comparison with the quadratic parabolic, triangular, trapezoidal, and other sections, it was desirable to develop a new open channel cross-section that has the smallest deviation from the semi-circular section.

In this research, different combinations of the exponent parameters ( $m$ and $n$ ) of the general parabolic section $\left(y=c x^{\mathrm{m} / \mathrm{n}}\right)$ were evaluated for $\mathrm{m}=1-5$ and $\mathrm{n}=1-20$. The results show that the discharge of the MEH section is maximum when $m=10$ and $n=3$. Therefore, a 
new open channel section called three and one-third (10/3) parabolic section is proposed.

Clearly, this new section type can enrich the simple family of parabolic sections.

\section{Characteristics of Proposed 10/3 Parabolic Section}

\subsection{Flow Area and Wetted Perimeter}

The geometry of the $10 / 3$ parabolic section is shown in Fig. 1 . Let $B$ be the water surface width and $h$ be the water depth. Then, the function of the proposed section is given by

$$
y=c\left|x^{10 / 3}\right|
$$

where $c=$ shape factor of the $10 / 3$ parabolic section, $y=$ vertical axis $(\mathrm{m})$, and $x=$ horizontal axis (m). Since $y=h$ at $x=B / 2$, then the relationship between $c, h$, and $B$ is given by

$$
c=8 \frac{\sqrt[3]{2} h}{B^{10 / 3}}
$$

where $B=$ water surface width (m) and $h=$ water depth (m). The flow area $A$ is obtained using integration as

$$
\begin{aligned}
A & \left.=2\left(\frac{B h}{2}-\int_{0}^{\frac{B}{2}} y \mathrm{~d} x\right)\right) \\
& =h B-\frac{32^{2 / 3} B^{13 / 3} c}{208}
\end{aligned}
$$

Substituting Eqs. (2) into (3), $A$ is expressed in terms of $h$ and $B$ as

$$
A=\frac{10 h B}{13}
$$

The variable $P$ can be derived by integration of the arc length as 
[5]

$$
\begin{aligned}
P & =2 \int_{0}^{\frac{B}{2}} d s=2 \int_{0}^{\frac{B}{2}} \sqrt{1+\left(\frac{d y}{d x}\right)^{2}} d x \\
& =2 \int_{0}^{\frac{B}{2}}\left(1 / 3 \sqrt{100 c^{2} x^{14 / 3}+9}\right) d x
\end{aligned}
$$

Clearly, it is difficult to compute the exact wetted perimeter using Eq. [5] and an approximation (presented later) is needed.

\subsection{Comparing Discharge Under the Same $B$ and $h$}

\section{Comparing with Square Parabola $\left(y=c x^{2}\right)$ Cross-Section}

The classic parabola section is defined as $y=c x^{2}$, where $A$ and $P$ are given in Monadjemi (1994). Assuming a dimensionless parameter $\omega_{2}=\frac{Q_{10 / 3}}{Q_{2}}$, then

$$
\begin{aligned}
& \omega_{2}=\frac{Q_{10 / 3}}{Q_{2}}=\frac{A_{10 / 3}{ }^{5 / 3} / P_{10 / 3}^{2 / 3}}{A_{2}^{5 / 3} / P_{2}^{2 / 3}} \\
& =\frac{\left(\frac{10 h B}{13}\right)^{5 / 3} /\left[2 \int_{0}^{\frac{B}{2}}\left(1 / 3 \sqrt{100 c^{2} x^{14 / 3}+9}\right) d x\right]^{2 / 3}}{\left(\frac{2}{3} B h\right)^{5 / 3} /\left[1 / 8 \frac{\ln \left(4 h+\sqrt{B^{2}+16 h^{2}}\right) B^{2}-\ln (B) B^{2}+4 \sqrt{B^{2}+16 h^{2}} h}{h}\right]^{2 / 3}}
\end{aligned}
$$

where $A_{2}=$ flow sectional area $\left(\mathrm{m}^{2}\right), P_{2}=$ wetted perimeter $(\mathrm{m})$ of $y=c x^{2}$ section, $Q_{2}=$ discharge under the same $B$ and $h\left(\mathrm{~m}^{3} / \mathrm{s}\right)$.

The variable $\omega_{2}$ is verified numerically with $h=0.01-20 \mathrm{~m}$ and $B=0.01-100 \mathrm{~m}$, with an increment of $0.01 \mathrm{~m}$. The results show that the minimum $\omega_{2}=1.220$, which means that the discharge of the proposed section is $22 \%$ larger than that of the quadratic parabolic section discharge.

Comparing with Cubic Parabola $\left(y=c x^{3}\right)$ Cross -Section 
The cubic parabola cross-section is defined as $y=c x^{3} \quad$ (Han and Said 2016). Assuming a dimensionless parameter which, $\omega_{3}=\frac{Q_{10 / 3}}{Q_{3}}$, which can been used to compare the discharge under the same $B$ and $h . \omega_{3}$ can be written as

$$
\begin{aligned}
& \omega_{3}=\frac{Q_{10 / 3}}{Q_{3}}=\frac{A_{10 / 3}^{5 / 3} / P_{10 / 3}^{2 / 3}}{A_{3}^{5 / 3} / P_{3}^{2 / 3}} \\
& =\frac{\left(\frac{10 h B}{13}\right)^{5 / 3} /\left[2 \int_{0}^{\frac{B}{2}}\left(1 / 3 \sqrt{100 c^{2} x^{14 / 3}+9}\right) d x\right]^{2 / 3}}{\left(\frac{3}{4} h B\right)^{5 / 3} /\left[2 \int_{0}^{B / 2} \sqrt{9 c^{2} x^{4}+1} \mathrm{~d} x\right]^{2 / 3}}
\end{aligned}
$$

where, $Q_{10 / 3}=$ flow discharge of the proposed section $\left(\mathrm{m}^{3} / \mathrm{s}\right)$ and $Q_{3}=$ discharge of cubic parabola section under the same $B$ and $h\left(\mathrm{~m}^{3} / \mathrm{s}\right) . \quad A_{3}=$ flow sectional area of the cubic parabola section $\left(\mathrm{m}^{2}\right)$, and $P_{3}=$ wetted perimeter for $y=c x^{3}$ section (m).

The variable $\omega_{3}$ is verified numerically with $h=0.01-20 \mathrm{~m}$ and $B=0.01-100 \mathrm{~m}$, with an increment of $0.01 \mathrm{~m}$. The results show that the minimum $\omega_{3}$ is also greater than one, which means that the discharge of the proposed section is larger than that of the quadratic parabola section discharge under the same $B$ and $h$.

The general section in the form $y=c x^{m / n}$ was evaluated for different combinations of $m$ and $n$, where $m=1-5$ and $n=1-20$. The results show the flow capacity of the proposed cross-section is larger than that of other parabola sections for given $B$ and $h$. 


\section{Practical Aspects}

\subsection{Wetted Perimeter Approximation}

There are several approximate methods for the integration of arc length. Here, the Gauss-Legendre integration method is used (Abramowitz and Stegun 1972). The three-point approximation is given by

$$
P=\frac{h^{3 / 10}(0.18519 \sqrt{9+0.0037642 J}+0.18519 \sqrt{9+57.235 J}+0.074074 \sqrt{144+62.996 J})}{c^{3 / 10}}
$$

where $J=c^{3 / 5} h^{7 / 5}$

Similarly, the four-point approximation is given by

$$
P=\frac{(0.11595 \sqrt{9+0.00039259 J}+0.21738 \sqrt{9+0.56640 J}+0.21738 \sqrt{9+15.428 J}+0.11595 \sqrt{9+71.476 J}) h^{3 / 10}}{c^{3 / 10}}
$$

For given $h$ and $B, c$ can been computed using Eq. [2].

To verify the accuracy of Eqs. (8) and (9), consider a 10/3 parabolic channel with shape factor $c=0.29$. It is required to compare the results of the explicit approximate formulas of Eqs. [8] and [9] for the wetted perimeter and those of numerical integration for $h=1.0-3.0 \mathrm{~m}$ (Table 1). The results indicate that the maximum relative error is $0.25 \%$ and $0.04 \%$ using the three and four-point approximate formulas, respectively. Thus, the approximate formulas are very accurate.

\subsection{Normal water depth calculation}

The discharge of the uniform flow of open channel can be written using Manning formula as (Chow 1959; Akgiray 2005; Easa 2011) 
[10]

$$
Q=\frac{1}{n} \frac{A^{5 / 3} i^{1 / 2}}{P^{2 / 3}}
$$

where $Q=$ flow discharge $\left(\mathrm{m}^{3} / \mathrm{s}\right), n=$ roughness of channel bed, $i=$ longitudinal grade of channel bottom.

Normal water depth can be computed by solving Eq. [10]. However this equation is a nonlinear equation and it is difficult to solving. Here, using the numerical fittings method, a simple iteration formula is proposed as

$$
h_{k+1}=0.65 \frac{Q^{0.6} n^{0.6} B^{0.4}\left(0.057976 \sqrt{9+0.000015457 a^{2} B^{14 / 3}}+W\right)^{0.4} a^{0.3}}{h^{0.3} i^{0.3}}
$$

where, $h_{k+1}=$ normal depth of the $k+1$ (th) times of iteration, $h=$ normal depth of the $k($ th)

times of iteration, $B=2 \frac{h^{3 / 10}}{a^{3 / 10}}$, and

$$
W=0.10869\left(\sqrt{9+0.022301 a^{2} B^{14 / 3}}+\sqrt{9+0.60745 a^{2} B^{14 / 3}}\right)+0.057976 \sqrt{9+2.8142 a^{2} B^{14 / 3}}
$$

As an example, for $Q=15 \mathrm{~m}^{3} / \mathrm{s}, n=0.014, c=0.2, i=1 / 11000$, using the numerical method $h$ $=4.31677 \mathrm{~m}$. Using the simple iteration formula of Eq. [11] with an initial value for $h=1.0 \mathrm{~m}$, then $h=4.317 \mathrm{~m}$ only after three iterations.

\section{Most Efficient Hydraulic Section}

\subsection{Model Structure of MEH Section}

The MEH maximizes $Q$ for given $A$ or $P$ or alternatively minimizes $A$ or $P$ for given $Q$. The case of given $P$ is represented by the following optimization model 
[13]

$$
\text { Maximize } Q=\frac{1}{n} \frac{A(h, B)^{5 / 3} i^{1 / 2}}{P(h, B)^{2 / 3}}
$$

$$
\text { Subject to } A(h, B)=C
$$

where, $C=$ constant flow area, $A=\frac{10 h B}{13}$

Substituting Eq. [14] into Eq. [13], the objective function will become,

$$
\operatorname{Maximize} \quad Q=\frac{1}{n} \frac{C^{5 / 3} i^{1 / 2}}{P(h, B)^{2 / 3}}
$$

Because $n, i$ and $C$ are constant value. This optimization model will become into

$$
\begin{array}{ll}
\text { Minimize } & P(h, B) \\
\text { Subject to } & \frac{10 h B}{13}=C
\end{array}
$$

\subsection{Solution of Model for MEH Section}

Although Eqs. (8) and (9) provide a simple and practical method for $P$ of the 10/3 parabolic section, they are approximate expressions. The partial derivatives of $P$ can be obtained by expressing the wetted perimeter $P$ of Eq. (5) using the super-geometric function as

$$
P=\frac{7 B}{10} H_{y}\left([3 / 14,1 / 2],\left[\frac{17}{14}\right],-\frac{400 h^{2}}{9 B^{2}}\right)+1 / 10 \sqrt{9 B^{2}+400 h^{2}}
$$

where $H_{y}=$ Hypergeom $([a, b], c, z)$, which is the super-geometric function

Assuming a dimensionless parameter, $\eta=\frac{B}{h}$ and after simplifying, Eq. [14] and Eq. [18] become

$$
h=1 / 10 \frac{\sqrt{130} \sqrt{\eta C}}{\eta}
$$




$$
P=1 / 10 h\left(7 \text { Hypergeom }\left([3 / 14,1 / 2],\left[\frac{17}{14}\right],-\frac{400}{9 \eta^{2}}\right) \eta+\sqrt{9 \eta^{2}+400}\right)
$$

Substituting Eq. [19] into Eq. [20], the optimization model (Eq. [16] and Eq. [17]) becomes

[21]Minimize $P=\frac{\sqrt{130} \sqrt{\eta C}}{100 \eta}\left(7\right.$ Hypergeom $\left.\left([3 / 14,1 / 2],\left[\frac{17}{14}\right],-\frac{400}{9 \eta^{2}}\right) \eta+\sqrt{9 \eta^{2}+400}\right)$

Clearly, Eq. [21] is equivalent to $\frac{\partial P}{\partial \eta}=0$. Then finding derivative of $P$ with respect to the variables $\eta$ and simplifying results in

$$
357 \sqrt{9 \eta^{2}+400} \eta^{2} H_{y 1}+459 \eta^{3}+5600 \sqrt{9 \eta^{2}+400} H_{y 2}-20400 \eta=0
$$

where

$$
H_{y 1}=\text { Hypergeom }\left([3 / 14,1 / 2],[17 / 14],-\frac{400}{9 \eta^{2}}\right), H_{y 2}=\text { Hypergeom }\left([17 / 14,3 / 2],[31 / 14],-\frac{400}{9 \eta^{2}}\right)
$$

Since $h>0$, solving Eq. [22] numerically yields the only and feasible solution as

$$
\eta=2.1273028
$$

This means that the exact ratio of water surface width to depth for the most efficient hydraulic section is 2.1273. It is a important parameter. Substituting $\eta=\frac{B}{h}=2.1273$ into Eq. [2], the most efficient hydraulic shape factor $c$ is obtained as

$$
c=0.8141 h^{-7 / 3}
$$

It should be noted that the same results can been obtained using Lagrange multipliers optimization method (Monadjemi 1994).

\subsection{Application Aspects of MEH Section}

Substituting for $\eta=\frac{B}{h}=2.1273$ into Eqs. [3] and [18], $A$ and $P$ are obtained as 


$$
A=1.6364 h^{2}
$$

$$
P=3.2298 h
$$

Substituting Eqs. [25] and [26] into Eq. [10] yields the following explicit formula for the discharge,

$$
Q=1.0400 \frac{h^{8 / 3} \sqrt{i}}{n}
$$

which can be applied to compute the discharge of the MEH section given the water depth.

From Eq. [27], the explicit formula for water depth is given by

$$
h=0.9854 \grave{o}^{3 / 8}
$$

where $\mathrm{o}=\frac{Q n}{\sqrt{i}}\left(\mathrm{~m}^{3} / \mathrm{s}\right)$.

The water depth can be computed using Eq. (24) given the discharge. Combining Eqs. [28] and [24], the explicit formula of the factor of the MEH section is obtained as

$$
c=0.8425 \mathrm{ò}^{-\frac{7}{8}}
$$

which can used to directly design the best hydraulic section using flow discharge.

\subsection{Explicit Formulas for Normal and Critical Water Depths}

The normal water depth is determined by Manning formula and is used in channel design, operation, flood control, and flow measurement and maintenance in open channels. Clearly, Eq. [28] can also be used for computing the normal water depth,

$$
h_{0}=0.9854 \hat{o}^{3 / 8}
$$


The critical water depth signifies the state of flow at which the specific energy is minimum for given $Q$. It represents the gradually varied flow into subcritical and supercritical flows, and plays an important role in analyzing important aspects such as water surface profile, hydraulic jump, and energy dissipation. The general formula of the critical water depth is given by

$$
\frac{\beta Q^{2}}{g}=\frac{A^{3}}{B}
$$

where $\beta=$ kinetic energy correction factor and $g=$ acceleration of gravity $\left(\mathrm{m} / \mathrm{s}^{2}\right)$.

Substituting for $B=2.1273 h$ and for $A$ from Eq. [25] into Eq. [31], the critical water depth is derives as following

$$
h_{c}=0.8654 \frac{\sqrt[5]{\beta} Q^{2 / 5}}{\sqrt[5]{g}}
$$

Example 2: Consider an open channel with $Q=26 \mathrm{~m}^{3} / \mathrm{s}, \quad n=0.014, i=1 / 9000$, and $\beta=1$. Design a 10/3 parabolic-shaped channel having maximum discharge. Substituting the known data into Eqs. [29] and [30], one obtains $c=0.038$ and $h=3.719 \mathrm{~m}$. Then, using Eqs. [23] and [33], one obtains $B=\eta h=7.912 \mathrm{~m}$ and $h_{c}=2.018 \mathrm{~m}$. From Eqs. [25] and [26], one gets $A=22.635 \mathrm{~m}^{2}$ and $P=12.012 \mathrm{~m}$.

\subsection{Comparison with Other Parabolic Sections (Under Optimal Conditions)}

For the 10/3 parabolic section, substituting Eqs. [28] into [25] and [26], $B, P$, and $A$ can be expressed in terms of $Q$ as 


$$
\begin{aligned}
& B=2.0963 \mathrm{o}^{3 / 8} \\
& P=3.1826 \mathrm{o}^{3 / 8} \\
& A=1.5890 \mathrm{o}^{3 / 4}
\end{aligned}
$$

where $\mathrm{o}=\frac{Q n}{\sqrt{i}}$.

Similarly, for the quadratic parabolic section $y=c x^{2}$, the optimal width-depth ratio can been obtained as $\eta=B / h=2.0555$ (Han 2015). The characteristics of the quadratic, cubic, semi-cubic parabolic, and semi-circle section are presented in Table 2.

As previously mentioned, to obtain the MEH section, the general section in the form $y=$ $c x^{m / n}$ was evaluated for different combinations of $m$ and $n$, where $m=1-5$ and $n=1-20$. The results show that the discharge of the MEH section is maximum when $m=10$ and $n=3$. This means that the parabola section $y=c x^{10 / 3}$ is the MEH section.

The results show that the water depth of the $10 / 3$ parabola section is smaller than that of the square parabola, cubic parabola and semi-cubic parabola section for given $Q$. In addition, comparison (Table 1) shows that $B, P$, and $A$ of the proposed section are smaller than those of other parabola section and trapezoidal sections for given $Q$. In other words the discharge of the 10/3 parabola section is larger than these of other parabola and trapezoidal sections too.

Comparison with the semi-circle section shows that the difference in the flow area between the 10/3 parabolic and semi-circular sections is very small. For example, for $Q=10$ $\mathrm{m}^{3} / \mathrm{s}, n=0.014$, and $i=1 / 10000, A=11.5003 \mathrm{~m}^{2}$ for the $10 / 3$ parabolic section and $A=$ $11.4590 \mathrm{~m}^{2}$ for the semi-circular section $\left(\right.$ difference $=0.0413 \mathrm{~m}^{2}$ ). However, the slope of the semi-circular section at the water surface is vertical which generally makes the construction of the freeboard rather difficult and exposes it to potential damaged. 


\section{Conclusions}

In this paper, a new so-called three and one-third parabolic channel section has been proposed.

For practical purposes, the section characteristics, including an explicit approximation for the wetted perimeter are presented, and a simple iteration algorithm to calculate the normal water depth is proposed. A model of the best hydraulic section along with its solution is developed. The results show that the width-depth ratio for the MEH section is 2.1273. Using this ratio, explicit formulas for the discharge, shape factor, normal depth, and critical depth are derived. The comparison results show that the proposed section has a larger discharge than the quadratic, cubic, and semi parabolic sections as well as the trapezoidal section. The hydraulic characteristics of the new section were found to be superior to those of other parabolic-shaped sections under optimal and non-optimal conditions.

\section{Acknowledgments}

This research project is supported by the Key Research and Development Program of Shandong Province, China (2016GSF117038), the National Science and Technology Support

Program of China (2015BAB07B02), the Development of Science and Technology Plan of Jinan City, China (201302052), and the Teaching \& Research Projects of the University of Jinan (J1641).

\section{REFERENCES}

Akgiray, O. 2005 Explicit solutions of the Manning equation for partially filled circular pipes. Can. J. Civ. Eng. 32: 490-499 (2005). doi: 10.1139/L05-001 
Aksoy, B. and Altan-Sakarya, A. 2006. Optimal lined channel design. Canadian Journal of Civil Engineering, 33(5): 535-545. doi: 10.1139/L06-008

Chahar, B. R. 2005. Optimal design of parabolic canal section. Journal of Irrigation and Drainage Engineering, 131(6): 546-554. doi: 10.1061/ASCE0733-94372005131:6546

Easa, S. M. 2016. Versatile general elliptic open channel cross section. KSCE Journal of Civil Engineering, 20(4): 1572-1581. doi:10.1007/s12205-015-0494-X

Easa, S. M. 2011. New and improved channel cross section with piece-wise linear or smooth sides. Canadian Journal of Civil Engineering, 38(6): 690-697. doi:10.1139/L11-037

Easa, S. M. 2009. Improved channel cross section with two-segment parabolic sides and horizontal bottom. Journal of Irrigation and Drainage Engineering, 135(6): 357-365. doi: 10.1061/ASCEIR.1943-4774.0000002

Han, Y-C. 2015. Horizontal bottomed semi-cubic parabolic channel and best hydraulic section. Flow Measurement and Instrumentation, 45: 56-61.

http://dx.doi.org/10.1016/j.flowmeasinst.2015.04.001

Han, Y-C and Easa, S.M. 2016. Superior cubic channel section and analytical solution of best hydraulic properties. Flow Measurement and Instrumentation, 50(2016): 169-177. http://dx.doi.org/10.1016/j.flowmeasinst.2016.06.019

Jain, A., Bhattacharjya, R.K., and Sanaga, S. 2004. Optimal design of composite channels using genetic algorithm. Journal of Irrigation and Drainage Engineering, 130(4): 286295. doi: 10.1061/(ASCE)0733-9437(2004)130:4(286)

Loganathan, J, G. 1991. Optimal design of parabolic canals. Journal of Irrigation and Drainage Engineering, 117(5): 716-735. 
Mironenko, A. P., Willardson, L. S., and Jenab, S. A. 1984. Parabolic canal design and analysis. Journal of Irrigation and Drainage Engineering, 110(2): 241-246.

Vatankhah, A.R. 2014. Semi-regular polygon as the best hydraulic section in practice (generalized solutions). Flow Measurement and Instrumentation, 38: 67-71.

http://dx.doi.org/10.1016/j.flowmeasinst.2014.05.016 


\section{LIST OF TABLES}

Table 1 Results of the wetted perimeter using approximate formulas

Table 2 The hydraulic characteristic of different shaped channel sections 
Table 1 Results of the wetted perimeter using approximate formulas

\begin{tabular}{cccccc}
\hline$h$ & $\begin{array}{c}\text { Theoretical } \\
P_{t} \\
(\mathrm{~m})\end{array}$ & $\begin{array}{c}\text { Three-Point } \\
\text { Formula } P_{3}\end{array}$ & $\begin{array}{c}\text { Absolute Error } \\
\left(P_{t}-P_{3}\right)\end{array}$ & $\begin{array}{c}\text { Four-Point } \\
\text { Formula } P_{4} \\
(\mathrm{~m})\end{array}$ & $\begin{array}{c}\text { Absolute } \\
\text { Error } \\
\left(P_{t}-P_{3}\right)\end{array}$ \\
\hline 1.0 & 3.840 & 3.838 & 0.002 & 3.840 & 0.000 \\
1.5 & 4.908 & 4.901 & 0.007 & 4.909 & 0.001 \\
2.0 & 5.951 & 5.939 & 0.012 & 5.953 & 0.002 \\
2.5 & 6.981 & 6.965 & 0.017 & 6.984 & 0.003 \\
3.0 & 8.004 & 7.984 & 0.020 & 8.007 & 0.003 \\
\hline
\end{tabular}


Table 2 The hydraulic characteristic of different shaped channel sections

\begin{tabular}{|c|c|c|c|c|c|}
\hline $\begin{array}{l}\text { Section } \\
\text { Type }\end{array}$ & $B / h$ & $\begin{array}{c}\text { Water Surface } \\
\text { Width, } B \\
\text { (m) }\end{array}$ & $\begin{array}{c}\text { Flow } \\
\text { Area, } A \\
\left(\mathrm{~m}^{2}\right)\end{array}$ & $\begin{array}{c}\text { Wetted } \\
\text { Perimeter, } P \\
\text { (m) }\end{array}$ & $\begin{array}{c}\text { Shape } \\
\text { Factor, } \\
a\end{array}$ \\
\hline Semi-circle & 2.0 & $2.00780^{3 / 8}$ & $1.58323 \mathrm{o}^{3 / 4}$ & $3.1539 \mathrm{o}^{3 / 8}$ & - \\
\hline $\begin{array}{l}\text { 10/3 } \\
\text { Parabolic }\end{array}$ & 2.127 & $2.0963 \mathrm{o}^{3 / 8}$ & $1.5890 \mathrm{o}^{3 / 4}$ & $3.1826 \mathrm{o}^{3 / 8}$ & $c=0.8425 \varepsilon^{-\frac{7}{8}}$ \\
\hline 3.0 Parabolic & 2.114 & $2.1165 \varepsilon^{3 / 8}$ & $1.5900 \varepsilon^{3 / 4}$ & $3.1847 \varepsilon^{3 / 8}$ & $c=0.8470 h^{-2}$ \\
\hline 2.0 Parabolic & 2.056 & $2.2213 \mathrm{o}^{3 / 8}$ & $1.6004 \mathrm{o}^{3 / 4}$ & $3.2401 \mathrm{o}^{3 / 8}$ & $c=0.9467 h^{-1}$ \\
\hline 3/2 Parabolic & 2.019 & $2.3355 \mathrm{o}^{3 / 8}$ & $1.6213 \mathrm{o}^{3 / 4}$ & $3.3469 \mathrm{o}^{3 / 8}$ & $c=0.9862 \frac{1}{\sqrt{h}}$ \\
\hline Trapezoidal & $2.309^{\mathrm{b}}$ & $2.2351 \hat{O}^{3 / 8}$ & $1.6224 \mathrm{o}^{3 / 4}$ & $3.3527 \mathrm{o}^{3 / 8}$ & $z=\sqrt{3} / 3$ \\
\hline
\end{tabular}

${ }^{\mathrm{a}} \mathrm{o}=Q n / \sqrt{i} . \quad{ }^{\mathrm{b}}$ the ration of bottom width to depth, $b / h=1.155$. 


\section{LIST OF FIGURES}

Fig. 1 Geometry of the 10/3 parabolic section 


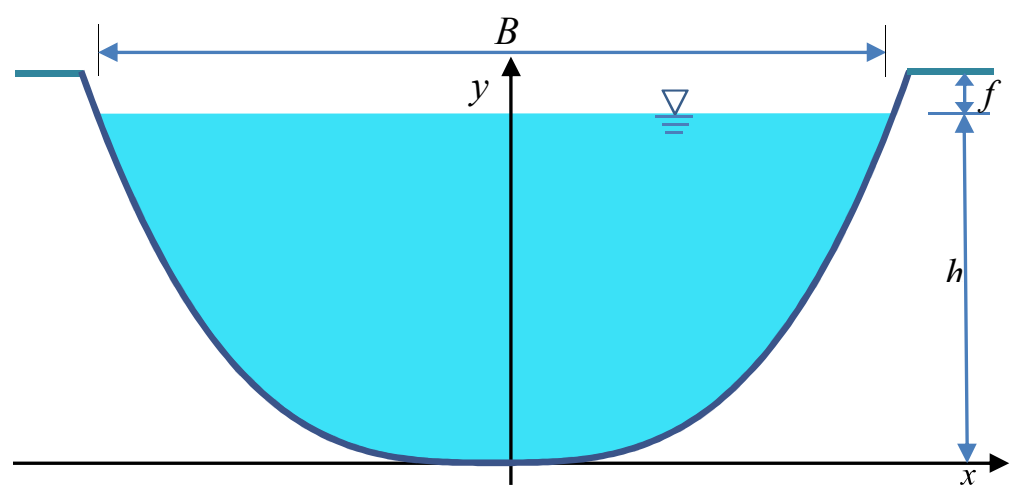

https://mc06.manuscriptcentral.com/cjce-pubs 